\title{
RING SOURCE POTENTIALS IN TWO SUPERPOSED FLUIDS SEPARATED BY AN INERTIAL SURFACE
}

\author{
B.N. MANDAL and KRISHNA KUNDU \\ Department of Applied Mathematics \\ Calcutta University \\ 92, A.P.C. Road, Calcutta - 700009 \\ INDIA
}

(Received May 23, 1986 and in revised form November 4, 1986)

ABSTRACT. The study of waves at the interface of two superposed fluids due to the presence of a vertical body of revolution requires the consideration of potentials due to horizontal ring sources submerged in one of the fluids. In this paper, the velocity potentials in the two fluids are computed due to a horizontal ring of sources of time-dependent strength submerged in either of the fluids of a two-fluid medium that are separated by an inertial surface.

KEYS WORDS AND PHRASES. Two-fluid medium, disconnected material, inertial surface, velocity potential, time-dependent strength, ring source.

1980 AMS SUBJECT CLASSIFICATION CODE. $76 B$.

1. INTRODUCTION.

The velocity potential due to a two-dimensional wave source of time-dependent strength submerged in a fluid of infinite depth with an inertial surface was obtained by Rhodes-Robinson [1]. Mandal and Kundu [2] extended this to the case of finite depth of the fluid and also obtained potentials due to other types of singularities present in the fluid.

Problems dealing with the generation of internal waves at the surface separating the two fluids due to the presence of a vertical body of revolution in either of the fluids can be formulated in terms of a suitable distribution of ring wave sources around the body as is done for a single fluid [3].

The potential due to a ring of wave sources of constant unit strength in an unbounded fluid has the form

$$
\phi_{0}(r, y)=2 \pi a \int_{0}^{\infty} \exp \{-k|y-n|\} J_{0}(k r) J_{0}(k a) d k
$$

where $a$ is the radius of the ring, $r$ is the distance from its axis, $y$-axis is taken as the axis of the ring [cf. [3]]. We note that $\phi_{0}$ is singular at points on the ring. Instead of an infinite fluid we now consider a fluid with a free surface or an inertial surface, or two superposed fluids separated by a horizontal plane which may or may not be an inertial surface where the ring source is submerged in either of the fluids. Then the resulting potential at any point will consist of $\phi_{0}$ 
together with an image potential due to the FS for a single fluid medium or due to the SS for a two fluid medium which is regular everywhere. This idea will be used here in obtaining the potentials due to a ring of sources.

In the present paper we obtain the velocity potentials due to a horizontal ring of wave sources of time-dependent strength submerged in one of the fluids of a twofluid medium separated by an inertial surface composed of a thin uniform distribution of disconnected materials. The upper fluid extends infinitely upwards while the lower fluid is of finite constant depth.

2. MATHEMATICAL FORMULATION OF THE PROBLEM.

We consider the irrotational motion of two inviscid incompressible fluids with $\rho_{1}$ and $\rho_{2} \quad\left(<\rho_{1}\right)$ as the densities of the lower and upper fluids respectively. The two fluids are separated by an inertial surface composed of a thin uniformly distributed disconnected material of area density $\left(\rho_{1}-\rho_{2}\right)$ under the action of gravity. The special case of an ordinary surface of separation corresponds to $\varepsilon=0$. The motion is due to a horizontal ring of wave sources of time-dependent strength submerged in either of the fluids.

We use a cylindrical co-ordinate system $(r, \theta, y)$ in which $y$-axis is taken vertically downwards and passes through the centre of a ring of wave sources each of strength $m(t)$ submerged in either of the fluids and the plane $y=0$ is the position of the inertial surface at rest. The lower fluid is of infinite horizontal extent and bounded by a horizontal bottom at some finite constant depth $h$ while the upper fluid is otherwise unbounded. We assume the motion starts from rest from the instant when the sources on the ring simultaneously start operating. Thus the motion is irrotational and can be described in each fluid by a corresponding potential function. The linearized description thus involves a pair of velocity potentials $\phi_{1}(r, \theta, y, t), \phi_{2}(r, \theta, y, t)$ which satisfy Laplace's equation in lower and upper fluid regions respectively except at points on the ring in the appropriate situation. The linearized kinematic equations are

$$
\frac{\partial \phi_{1}}{\partial y}=\frac{\partial \phi_{2}}{\partial y}=\frac{\partial \eta}{\partial t} \quad \text { on } \quad y=0
$$

where $n(r, \theta, t)$ denotes the inertial surface depression below the mean position. Since the surface of separation of the two fluids is covered with disconnected particles of surface density $\left(\rho_{1}-\rho_{2}\right) \varepsilon$ Newton's equation of motion for one of these particles is then

$$
\left(\rho_{1}-\rho_{2}\right) \varepsilon \ddot{\eta}=\rho_{2}\left(-\frac{\partial \phi_{2}}{\partial t}+g \eta\right)-\rho_{1}\left(-\frac{\partial \phi_{1}}{\partial t}+g \eta\right)+\left(\rho_{1}-\rho_{2}\right) \varepsilon \text { on } y=0
$$

By eliminating $\eta$ in (2.1) and (2.2) we obtain

$$
\frac{\partial^{2}}{\partial \mathrm{t}^{2}}\left(\phi_{1}-\varepsilon \frac{\partial \phi_{1}}{\partial \mathrm{y}}\right)-\mathrm{g} \frac{\partial \phi_{1}}{\partial \mathrm{y}}=\mathrm{s}\left\{\frac{\partial^{2}}{\partial \mathrm{t}^{2}}\left(\phi_{2}-\varepsilon \frac{\partial \phi_{2}}{\partial y}\right)-\mathrm{g} \frac{\partial \phi_{2}}{\partial \mathrm{y}}\right\} \text { on } \mathrm{y}=0
$$

Also the bottom and top conditions satisfled by $\phi_{1}$ and $\phi_{2}$ resepctively are 


$$
\frac{\partial \phi_{1}}{\partial y}=0 \text { on } y=h
$$

and

$$
\nabla \phi_{2} \rightarrow 0 \text { as } \mathrm{y} \rightarrow-\infty
$$

It may be noted that for a ring source with the time harmonic strengths of the same angular frequency $\sigma$ the inertial SS condition (2.3) becomes

$$
K \phi_{1}+(1-K \varepsilon) \frac{\partial \phi_{1}}{\partial y}=s\left\{K \phi_{2}+(1-K \varepsilon) \frac{\partial \phi_{2}}{\partial y}\right\} \text { on } y=0 \text {, }
$$

where $K=\frac{\sigma^{2}}{g}$. For $0 \leqq K \varepsilon<1$ the form of (2.5) is

$$
K * \phi_{1}+\frac{\partial \phi_{1}}{\partial y}=s\left(K * \phi_{2}+\frac{\partial \phi_{2}}{\partial y}\right) \text { on } y=0 \text {, }
$$

where $K^{*}=K(1-\varepsilon K)^{-1}$. This is merely a modification of the usual ss condition [4] corresponding to $\varepsilon=0$. However as noted in [1], for $K \varepsilon \geq 1$ the condition (2.5) does not allow propagation of time-harmonic progressive waves.

Let $\bar{\phi}_{i}(r, \theta, y, p)$ denote the Laplace transform of $\phi_{i}(r, \theta, y, t)(i=1,2)$ defined as

$$
\bar{\phi}_{1}=\int_{0}^{\infty} \exp (-p t) \phi_{1} d t \quad(p>0),
$$

then $\bar{\Phi}_{i}^{\prime}$ 's $(i=1,2)$ satisfy the BVP described by

$$
\begin{array}{ll}
\nabla^{2} \bar{\phi}_{1}=0, & y>0 \\
\nabla^{2} \bar{\phi}_{2}=0, & y<0
\end{array}
$$

except at points on the ring in the appropriate situation,

$$
\begin{gathered}
\frac{\partial \bar{\phi}_{1}}{\partial \mathrm{y}}=\frac{\partial \bar{\phi}_{2}}{\partial \mathrm{y}} \text { on } \mathrm{y}=0, \\
\mathrm{p}^{2} \bar{\phi}_{1}-\left(\mathrm{g}+\varepsilon \mathrm{p}^{2}\right) \frac{\partial \bar{\Phi}_{1}}{\partial \mathrm{y}}=\mathrm{s}\left\{\mathrm{p}^{2} \bar{\phi}_{2}-\left(\mathrm{g}+\varepsilon \mathrm{p}^{2}\right) \frac{\partial \bar{\Phi}_{2}}{\partial \mathrm{y}}\right\} \quad \text { on } \mathrm{y}=0, \\
\frac{\partial \bar{\phi}_{1}}{\partial \mathrm{y}}=0 \text { on } \mathrm{y}=\mathrm{h}, \\
\left|\nabla \bar{\phi}_{2}\right| \rightarrow 0 \text { as } \mathrm{y} \rightarrow-\infty
\end{gathered}
$$

$\bar{\phi}_{i}(i=1,2)$ can be obtained in a manner analogous to the corresponding time-harmonic problems in a two-fluid medium. Laplace inversion will then give $\phi_{i}(i=1,2)$.

3. RING SOURCE SUBMERGED IN LOWER FLUID.

(i) Ring Source. Let the centre of the ring source be at a distance $f$ from the mean inertial SS and the radius of the ring be a. In this case $\bar{\phi}_{1}$, $\bar{\phi}_{2}$ are solutions of the BVP stated above with

$$
\bar{\phi}_{1} \rightarrow \bar{m}(p) \phi_{0} \text { as }\left\{(r-a)^{2}+(y-f)^{2}\right\}^{\frac{1}{2}} \rightarrow 0 \text {. }
$$


where $\bar{m}(p)$ is the Laplace transform of the time-dependent strength $m(t)$. We can write

$$
\begin{aligned}
\bar{\phi}_{1} & =\overline{\mathrm{m}} \phi_{0}-2 \pi a \overline{\mathrm{m}} \int_{0}^{\infty} \exp \{-\mathrm{k}(\mathrm{y}+n)\} \mathrm{J}_{\mathrm{o}}(\mathrm{kr}) \mathrm{J}_{\mathrm{o}}(\mathrm{ka}) \mathrm{dk} \\
& +\int_{0}^{\infty}\{\mathrm{A}(\mathrm{k}) \cosh \mathrm{k}(\mathrm{h}-\mathrm{y})+\mathrm{B}(\mathrm{k}) \sinh \mathrm{ky}\} \mathrm{J}_{\mathrm{o}}(\mathrm{kr}) \mathrm{J}_{\mathrm{o}}(\mathrm{ka}) \mathrm{dk}, \\
\bar{\phi}_{2} & =\int_{0}^{\infty} \mathrm{C}(\mathrm{k}) \exp (\mathrm{ky}) \mathrm{J}_{\mathrm{o}}(\mathrm{kr}) \mathrm{dk},
\end{aligned}
$$

where $A(k), B(k), C(k)$ are to be obtained by using the two SS conditions (2.8) and (2.9) and the bottom condition (2.10).

The final results after rearrangement take the forms

$$
\begin{aligned}
& \bar{\phi}_{1}=\bar{m}\left[U(r, \theta, y)+4 \pi a \int_{0}^{\infty} \frac{\cosh k(h-y) \cosh k(h-\eta)}{D(k) \sinh k h} J_{0}(k r) J_{0}(k a) \frac{\mu^{2}}{\mu^{2}+p^{2}} d k\right], \\
& \bar{\phi}_{2}=\bar{m}\left[V(r, \theta, y)-4 \pi a \int_{0}^{\infty} \frac{\exp (k y) \cosh k(h-\eta)}{D(k)} J_{0}(k r) J_{0}(k a) \frac{\mu^{2}}{\mu^{2}+p^{2}} d k\right],
\end{aligned}
$$

where

$$
\begin{aligned}
& U(r, \theta, y)=2 \pi a\left[\int_{0}^{\infty}[\{\exp (-k|y-n|)-\exp (-k|y+n|)\}\right. \\
&+\frac{2\{s(1-k \varepsilon)+k \varepsilon\}}{D(k) \cosh k h} \cosh k(h-y) \cosh k(h-n) \\
&\left.+\frac{2 \exp (-k h) \sinh k n \sinh k y}{\cosh k h} J_{0}(k r) J_{0}(k a) d k\right] \\
& V(r, \theta, y)=4 \pi a \int_{0}^{\infty} \frac{\exp (k y) \cosh k(h-n)}{D(k)} J_{0}(k r) J_{0}(k a) d k \\
& D(k)=\cosh k h+\{s(1-k \varepsilon)+k \varepsilon\} \sinh k h \\
& \mu^{2}=g k(1-s) \sinh k h / D(k) .
\end{aligned}
$$

Laplace inversion then gives

$$
\begin{aligned}
& \phi_{1}=m(t) U+4 \pi a \int_{0}^{\infty} \frac{\mu \cosh k(h-y) \cosh k(h-\eta)}{D(k) \sinh k h} J_{0}(k r) J_{0}(k a) \int_{0}^{t} m(\tau) \sin \mu(t-\tau) d \tau d k, \\
& \phi_{2}=m(t) v-4 \pi a \int_{0}^{\infty} \frac{\mu \exp (k y) \cosh k(h-\eta)}{D(k)} J_{0}(k r) J_{0}(k a) \int_{0}^{t} m(\tau) \sin \mu(t-\tau) d \tau d k(3.5)
\end{aligned}
$$

Now we consider three particular forms of the ring source strength, viz. it is

i) impulsive in nature at the initial instant but otherwise zero,

ii) constant in nature and

iii) time-harmonic in nature. 
For the impulsive case $m(t)=\phi(t)$, and the corresponding potentials are

$\phi_{1}{ }^{(0)}=\delta(t) U+4 \pi a \int_{0}^{\infty} \frac{\mu \cosh k(h-\eta) \cosh k(h-y)}{D(k) \sinh k h} J_{0}(k r) J_{0}(k a)$ sin $\mu t d k$,

$\phi_{2}{ }^{(0)}=\delta(t) V-4 \pi a \int_{0}^{\infty} \frac{\mu \exp (k y) \cosh k(h-\eta)}{D(k)} J_{0}(k r) J_{0}(k a) \sin \mu t d k$.

The potentials for the classical ring source of constant strength $m(t)=1$ are

$\phi_{1}{ }^{(1)}=U+4 \pi a \int_{0}^{\infty} \frac{\cosh k(h-\eta) \cosh k(h-y)}{D(k) \sinh k h}(1-\cos \mu t) J_{0}(k r) J_{0}(k a) d k$,

$\phi_{2}{ }^{(1)}=v-4 \pi a \int_{0}^{\infty} \frac{\exp (k y) \cosh k(h-\eta)}{D(k)} J_{0}(k r) J_{0}(k a)(1-\cos \mu t) d k$

For time-harmonic case $m(t)=$ sin $\sigma t$, and the velocity potentials are then given by $\phi_{1}^{(2)}=\sin \sigma t U+4 \pi a \int_{0}^{\infty} \frac{\mu \cosh k(h-\eta) \cosh k(h-y)}{D(k) \sinh k h} J_{0}(k r) J_{0}(k a) \frac{\mu \sin \sigma t-\sigma \sin \mu t}{\mu^{2}-\sigma^{2}} d k(3.8)$ $\phi_{2}^{(2)}=\sin \sigma t \mathrm{~V}-4 \pi a \int_{0}^{\infty} \frac{\mu \exp (k y)}{D(k)} \cosh k(h-n) J_{0}(k r) J_{0}(k a) \frac{\mu \sin \sigma t-\sigma \sin \mu t}{\mu^{2}-\sigma^{2}} d k$. Now to obtain the forms of these latter potentials for large time, following [1] we introduce in the integrals involving sin $\mu t$ a Cauchy principal value $k^{*} k_{0}^{*}$ which is the positive zero of $\Delta(k)$ where $\Delta(k)=\{k(1-s)(1-K \varepsilon)-K s\} s i n h k h-K \cosh k h$ when $0 \leqq K \varepsilon<1$.

Hence as $t \rightarrow \infty$ using the Riemann Lebesgue Lemma as in [1] we obtain after simplification

$$
\begin{aligned}
& \phi_{1}^{(2)} \sim 2 \pi a \sin \sigma t\left[\int_{0}^{\infty}\{\exp (-k|y-n|)-\exp (-k|y+n|)\} J_{0}(k a) J_{0}(k r) d k\right. \\
& +2 \int_{0}^{\infty} \exp (-k h) \sinh k n \sinh k y \frac{J_{0}(k a)}{\cosh k h} J_{0}(k r) d k+ \\
& \left.+2 \int_{0}^{\infty} \frac{k(1-s)(1-K \varepsilon)-K s \cosh k(h-\eta) \cosh k(h-y) J_{0}(k a) J_{0}(k r) d k}{\Delta(k) \cosh k h}\right] \\
& -\cos \text { ot } \frac{8 \pi^{2} a k_{0}^{*} \cosh k_{0}^{*}(h-\eta) \cosh k_{0}^{*}(h-y) J_{0}\left(k_{0}^{*} a\right) J_{0}\left(k_{0}^{*} r\right)}{\sinh 2 k_{0}^{*} h+s \cosh 2 k_{0}^{*} h+2 k_{0}^{*} h-2 s}, r>a, \\
& \phi_{2}^{(2)} \sim-4 \pi a k \sin \sigma t \int_{0}^{\infty} \frac{\exp (k y)}{\Delta(k)} \cosh k(h-\eta) J_{0}(k a) J_{0}(k r) d k \\
& +\cos \sigma t \frac{8 \pi^{2} a k_{0}^{*} \exp \left(k_{0}^{*} y\right) \cosh k_{o}^{*}(h-n) J_{o}\left(k_{o}^{*} a\right) J_{o}\left(k_{o}^{*} r\right)}{\sinh 2 k_{0}^{*} h+s \cosh 2 k_{0}^{*} h+2 k_{0}^{*} h-2 s}, r>a,
\end{aligned}
$$

These have the usual outgoing behavoiurs as $r+\infty$.

When $K \varepsilon \geq 1$, there is no zero of $\Delta(k)$ for $k>0$. Then by Riemann-Lebesgue lemma the integrals involving sin $\mu \mathrm{t}$ in (3.8) are wholly transient and after 
simplification we obtain

$$
\begin{aligned}
& \phi_{1}^{(2)} \sim 2 \pi a \sin \sigma t\left[\int_{0}^{\infty}\{\exp (-k|y-n|)-\exp (-k|y+n|)\} J_{0}(k a) J_{0}(k r) d k\right. \\
& +2 \int_{0}^{\infty} \exp (-k h) \sinh k n \sinh k y \frac{J_{0}(k a)}{\cosh k h} J_{0}(k r) d k \\
& \left.-2 \int_{0}^{\infty} \frac{\{k(1-s)(k \varepsilon-1)+k s\} \cosh k(h-n) \cosh k(h-y) J_{0}(k a) J_{0}(k r) d k}{\Delta(k) \cosh k h}\right] \\
& \phi_{2}^{(2)} \sim-4 \pi a k \sin \sigma t \int_{0}^{\infty} \frac{\exp (k y) \cosh k(h-n) J_{0}(k a) J_{0}(k r)}{\Delta(k)} d k .
\end{aligned}
$$

(ii) Ring Source Submerged in Upper Fluid. In this case the centre of the ring is situated at the point $(0,0,-f)$ in the upper fluid. Thus the boundary value problem for $\bar{\phi}_{1}$ and $\bar{\phi}_{2}$ in this case is given be equations (2.7) to (2.10) supplemented by the condition

$$
\bar{\phi}_{2} \rightarrow \bar{m} \phi_{0} \text { as }\left\{(r-a)^{2}+(y+f)^{2}\right\}^{\frac{1}{2}} \rightarrow 0
$$

For solution we try as

$$
\begin{gathered}
\bar{\phi}_{1}=\overline{\mathrm{m}} \int_{0}^{\infty}\{\mathrm{A}(\mathrm{k}) \cosh \mathrm{k}(\mathrm{h}-\mathrm{y})+\mathrm{B}(\mathrm{k}) \sinh \mathrm{ky}\} \mathrm{J}_{\mathrm{o}}(\mathrm{kr}) \mathrm{J}_{\mathrm{o}}(\mathrm{ka}) \mathrm{dk}, \\
\bar{\phi}_{2}=\overline{\mathrm{m}} \phi_{0}-2 \pi \mathrm{a} \overline{\mathrm{m}}_{0}^{\infty} \exp \{-\mathrm{k}(\mathrm{y}+n)\} \mathrm{J}_{\mathrm{o}}(\mathrm{kr}) \mathrm{J}_{\mathrm{o}}(\mathrm{ka}) \mathrm{dk}+\int_{0}^{\infty} \mathrm{C}(\mathrm{k}) \exp (\mathrm{ky}) \mathrm{J}_{\mathrm{o}}(\mathrm{kr}) \mathrm{dk} .
\end{gathered}
$$

Then as in case (i) we obtain

$$
\begin{gathered}
\phi_{1}=m(t) W(r, \theta, y)+4 \pi a s \int_{0}^{\infty} \frac{\mu \exp (-k n) \cosh k(h-y)}{D(k)} \\
J_{0}(k r) J_{0}(k a) \int_{0}^{t} m(\tau) \sin \mu(t-\tau) d \tau d k, \\
\phi_{2}=m(t) Z-4 \pi a s \int_{0}^{\infty} \frac{\mu \exp \{k(y-\eta)\} \operatorname{sinh~kh}}{D(k)} \\
\quad J_{0}(k r) J_{0}(k a) \int_{0}^{t} m(\tau) \sin \mu(t-\tau) d \tau d k,
\end{gathered}
$$

where

$$
\begin{aligned}
W(r, \theta, y) & =-4 \pi a s \int_{0}^{\infty} \frac{\exp (-k n)}{D(k)} \cosh k(h-y) J_{0}(k r) J_{0}(k a) d k \\
Z(r, \theta, y) & =2 \pi a\left[\int_{0}^{\infty}\{\exp (-k|y-n|)-\exp (-k|y+n|)\} J_{0}(k r) J_{0}(k a) d k\right. \\
& \left.-2 \int_{0}^{\infty} \frac{\exp \{k(y-n)\}}{D(k)}\{\cosh k h+k \varepsilon(1-s) \sinh k h\} J_{0}(k r) K_{0}(k a) d k\right]
\end{aligned}
$$


For different source strengths the potentials for the ring source can be calculated as in the prevoius case (i).

4. DISCUSSION.

Ring source potentials present in one of the fluids of a two-fluid medium separated by an inertial surface are obtained for time-dependent source strengths. The effect of interfacial tension at the surface of separation is neglected. Known results for the one-fluid medium can be made evident by substituting $s=0$. Putting $s=0$ and $\varepsilon=0$ simultaneously in (3.9) the result can be identified with that obtained in [5]. The results however can be extended to include the effect of surface tension at the surface of separation.

ACKNOWLEDGEMENT. One of the authors (KK) thanks the Calcutta University for the award of U.G.C. fellowship.

\section{REFERENCES}

1. RHODES-ROBINSON, P.F. On the Generation of Water Waves at an Inertial Surface, J. Austral. Math. Soc. Ser B 25 (1984), 366-383.

2. MANDAL, B.N. and KUNDU, K. A Note on the Singularities in the Theory of Water Waves with an Inertial Surface, J. Austral. Math, Soc. Ser B (1986), to appear.

3. HULME, A. The Potential of a Horizontal Ring of Wave Sources in a Fluid with a Free Surface, Proc. Roy. Soc. Lond A 375(1981), 295-305.

4. GORGUI, M.A. and KASSEM, S.E. Basic Singularities in the Theory of Internal Waves, Q. J1. Mech. App1. Math. 31 (1978), 31-48.

5. RHODES-ROBINSON, P.F. On the Surface Waves in the Presence of Immersed Vertical Boundaries I, Q. J1. Mech. App1. Math. 32(1979), 109-124. 


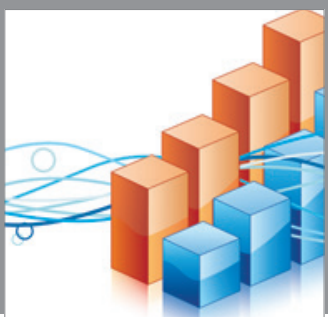

Advances in

Operations Research

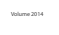

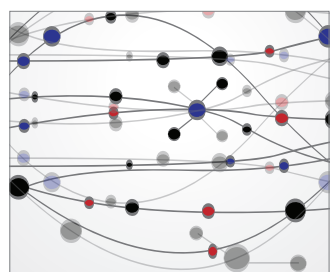

\section{The Scientific} World Journal
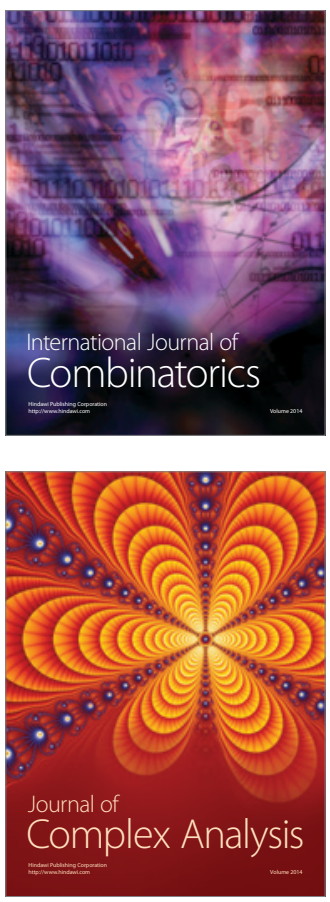

International Journal of

Mathematics and

Mathematical

Sciences
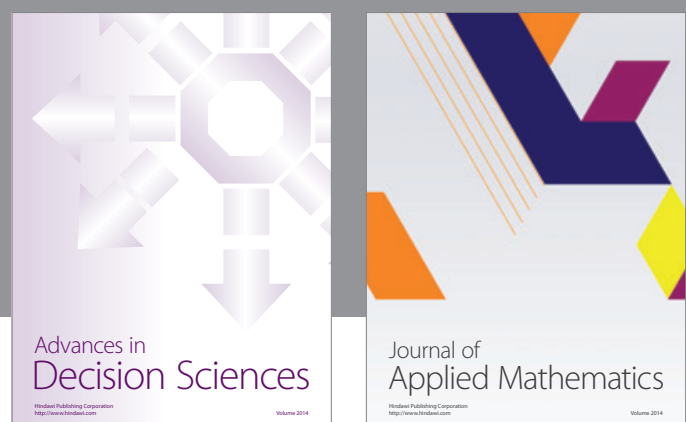

Journal of

Applied Mathematics
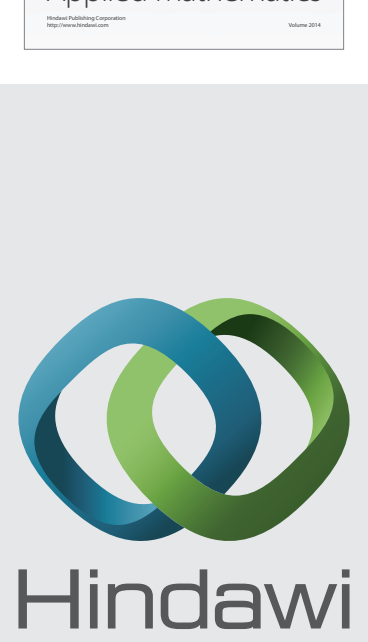

Submit your manuscripts at http://www.hindawi.com
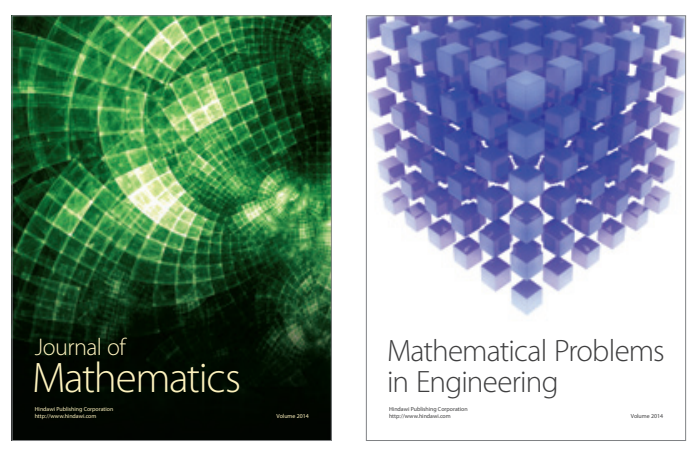

Mathematical Problems in Engineering
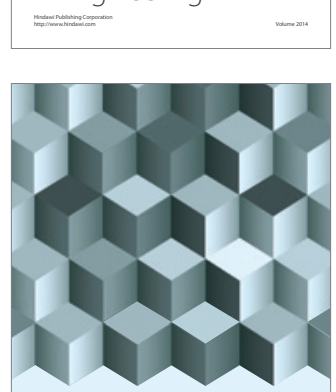

Journal of

Function Spaces
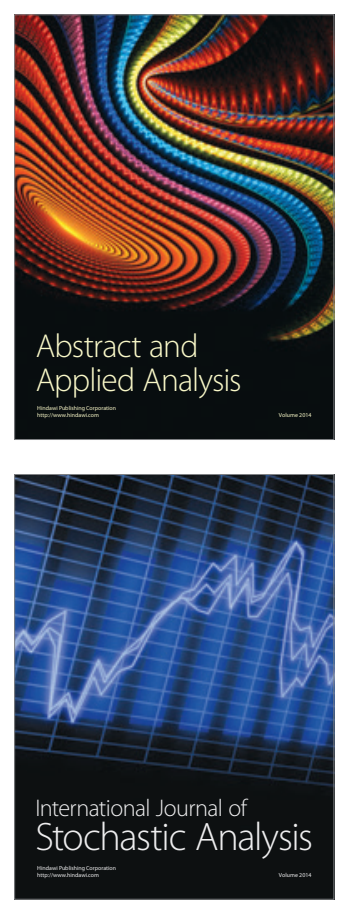

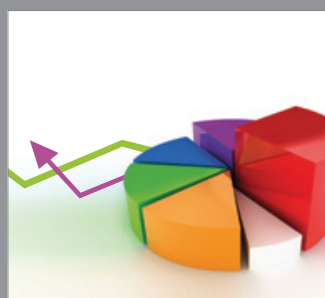

ournal of

Probability and Statistics

Promensencen
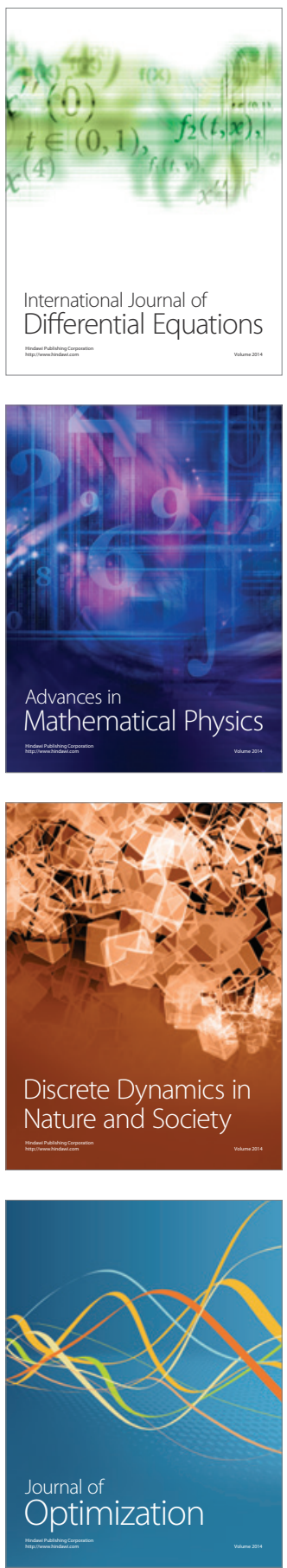